\title{
Summary of three-dimensional animation creation based on ethnic culture element
}

\author{
Zhaopo Shao \\ School of Fine Arts, Huanggang Normal University, Huanggang, Hubei, China
}

\begin{abstract}
It is an artistic expression form combining painting, film \& television, digital technology, music, and literature. As an audio and visual art, three-dimensional animation has its own unique culture-loading function, technical aesthetic characteristics, and requirements for national art expression. This paper aims to find the method to combine digital technology and national art in combination of three-dimensional animation short film creation, and hopes to clear the road for the cultivation of domestic three-dimensional animation quality project.
\end{abstract}

Keywords: three-dimensional animation; digital technology; national art

Gustave Flaubert, a French author, once said, "The longer art develops, the more scientific it will be; in the meantime, science will be artistic. These two will break up on the basis and converge on the top at last." With the popularization of globalization and culture, all movie historians accept that contemporary film industry couldn't have created current scale if there were no workers for film test who have made continuous contribution to promoting the possibility of videos to an extreme. In recent years, more and more digital technologies have been applied in animation production and film making with the development of computer hardware and software. Due to the help in making frames fine, effect vivid and creation convenient, digital technologies have won a large number of audiences and favor from more digital artists. However, we cannot neglect the problems of low quality, blind pursuit for technical content and absence of artistic research on animation in animation works. Therefore, if domestic animation plans to keep a foothold in the world, intensive study should be made on the relations between animation technology and national art.

\section{AESTHETIC CHARACTERISTICS OF THREE-DIMENSIONAL ANIMATION TECHNOLOGY}

1) Technical characteristics
Digital three-dimensional animation is an animation art that requires high technical content and needs further development and exploration in creation language. Technology is a specific method to reach specific goals. It is the principal part of knowledge evolution. So is the development of animation production technique. Animation is a product of technical advance. Without technical advance, no animation can be created. During the early stage of animation, how to create a technology to make "frames move" is the main problem for animation artists. Solutions were all about how to make static "frames" move until animation production technology appeared in 1915. With the improvement of animation production technology, how to make animation "move" is no longer a problem. As computer information technology is in continuous development, three-dimensional animation has occupied the dominant position. Production of three-dimensional animation film cannot exist without CG digital technology. CG is short for Computer Graphics. ${ }^{[3]}$ The exploration to produce animation by using computer graphics technology started in early 1980s. At that time, the production of three- dimensional animation was mainly completed in large-scale work stations. 3D Studio software was in absolute monopoly position when all PC run in DOS operating system. In 1994, Microsoft launched Windows operating system and moved Softimage from working station to PC. In 1995, Win95 appeared and 3DS MAX1.0 replaced 3DS. In 1998, the emergence of 
Maya can be called another milestone in three- dimensional animation development history. Maya is a world's top three-dimensional animation software produced by American Autodesk Company. It can be applied in professional film and television advertisement, character animation, and special effects of motion pictures. Maya is improved in function, flexible in working, easy in learning and use, high in producing efficiency, and strong in reality sense rendering. It is high-end manufacturing software in film level. The three-dimensional animation mentioned here refers to the newly-developing production method of using MAYA software to finish the rendering procedure and create image motions according to the virtual scenes, characters, textures, modulation motions, and setting lights shown in stories and original drawings. It can be seen that there are strong characteristics of the times contained in animation technology. Rapid development in technology can help animation artists free their subjective artistic performance and create better animation works.

\section{2) Aesthetic styles of image}

Three-dimensional animation can manifest perfect spatial shape creation and real texture. More freedom is allowed in three-dimensional animation creation. The virtual lens and lighting can truly realize animation artist's subjective ideas. In 1995, PIXAR Computer Art Studio completed Toy Story which is the first three-dimensional animation full of stereoscopic impression and sense of reality created by digital technology. It is an important turning point in the world animation history. Later, it only took threedimensional animation less than 10 years to occupy the leading role in animation creation industry. Threedimensional animation films, such as Monsters Inc., Finding Nemo, and UP, have won the Short Film Award in Oscar and made new records in the world box office history. Seen from the outside, it seems the sensation caused by these three-dimensional animation films is a reform of audience's visual enjoyment. However, the root cause lies in the fact that each three-dimensional animation film has developed a new producing technology to make an obvious reform in visual effect. The emergence of three-dimensional animation has made the room for two-dimensional animation so much less that Walt Disney Company had to offer a high price for acquiring Pixar which has always focused on three-dimensional animation, in order to return to its leading role in animation industry. There is no doubt that aesthetic style of image is in continuous progress and has promoted the development of animation technology to a brand new area.

\section{ARTISTRY IS THE SOUL OF THREE- DIMENSIONAL ANIMATION CREATION}

As a comprehensive art, animation is an artistic presentation form in combination of painting, film, digital media, music, and literature. Image is in the core of Chinese traditional aesthetics. It reflects the good wishes manifested by social life and idea expression. Script is the foundation of animation literariness. The production of an animation film starts from creating an animation script. After the animation script created by technical means is confirmed, the creator can draw the frames of story boards as the previews of the future film. Script is not only the important proof for further designs and construction schemes; but is also the guiding blueprint of original drawing and background mapping. Many Chinese animation artists in older generation have created world-renowned animation works, such as Havoc in Heaven, Prince Nezha's Triumph against Dragon King, and Little Tadpole Looking for Mummy which is a cartoon film in Chinese ink and water colors. All these works contain strong artistic appeal and have been praised as "Chinese animation school" by animation artists all over the world. The cartoon film Kung Fu Panda made by Dreamworks was well received after being on show in cinemas around the world. All the things shown in this film come from China. Why can't we make such a good story? John Stevenson, the director of Kung Fu Panda, has studied Chinese culture for three decades. Animation modeling includes character modeling, scene design, and tool design. Design of animation modeling is a process of artistic creation. Besides, it is also a process of broad reference and absorption. Artists use exaggeration and deformation to process the characters and their personalities and personal charm based on rich living experience and skillful modeling techniques. Artists use animation characters to reflect people's subjective wishes. All character creation comes from life. Any character with no base in life cannot arouse people's love and spiritual resonance.

\section{METHODS TO COMBINE DIGITAL TECHNOLOGY AND NATIONAL ART}

The Horse, a short three-dimensional animation film, has won many awards, such as The Best Story Award in 2010 Changchun International Digital Entertainment Animation Art Festival, and Excellent Animated Short Film awarded by Beijing Film Academy. The writer will make analysis of the methods to combine digital technology and national art based on his own production process as follows.

\subsection{Technical characteristics of story content}

The Horse has strong literariness with compact story structure and gradual development of paradox and suspense. It contains the basic conditions to produce a high-quality animation film. The Horse tells a story between a county magistrate and an ordinary citizen in 
ancient time. By describing the process of how the magistrate stole the horse from a farmer's home, it has many settings in details. The story begins with the magistrate's greedy image. After a yamen runner tells the magistrate there's a precious horse in a farmer's home, the magistrate decides to steal it. The short film focuses on the process of the magistrate's horse stealing, such as the scene when the magistrate enters the farmer's home and hides himself after the farmer sees him. In the end, when the magistrate starts stealing the horse and fights with the horse, the climax of the whole story comes. The result of this story is the magistrate fails to steal the horse and gets kicked by it. The story line of The Horse is full of irony and entertainment. However, test of CG technology is the precondition to start producing this animation. If the technical problems existing in the film cannot be solved, the transition from abstract literal art to visual entity art cannot be achieved. For example, the creation of storyline and shooting tone, test of shot application, creation of the characters' emotion and personalities, motion test, skeleton setting, uniformity of weight production, performance exaggeration and lyricism, descriptive performance practice, effect of the blowing dust, and test of MAYA particle effects are the preconditions to ensure the smooth production of this animation film. Without these technical tests, the smooth transition from literal story to animation is impossible.

\subsection{Application of plastic arts in modeling of three-dimensional animation}

Modeling quality is critical in the quality of the entire animation. Original painting is the proof for modeling production. Model is the virtual presentation of the original painting accomplished by three-dimensional software MAYA. Animation character is one of the key factors for the success of an animation film. Cute cartoon modeling styles are usually used for character image design. As excessive realism may weaken the entertainment of this film, the prototype designs of the characters in this film are all in cute cartoon style. There are four characters in this animation work. Exaggeration and contrast are used in character design, especially in the designs of the county magistrate and his yamen runner. The magistrate is short and fat to show his greed while the yamen runner is tall and thin to constitute obvious contrast to the magistrate. The yamen runner is in simple and unadorned clothes while the patterns on the magistrate's clothes are same as the patterns and tones of ancient official's robe. Ancient farmer's poverty is referred to in the design of the farmer so as to show his poverty in an exaggerated way. The precious horse is in white with a long neck, heavy horse mane, strong skeleton, and a powerful tail. The horse can give a presentative feeling and show its unique feature to whoever sees it. (See Figure 1 for character image designs)

Before modeling, the personalities, shapes and other details of the characters in this story must be fully understood. Three-dimensional models should only be produced after getting familiar with these preconditions. The first two problems are the modeling structures of the original paintings and the arrangement of the three-dimensional models. Model arrangement is around character structure. The modeling lines move with the structure. Thus, detailed description about structural points must be given by the original painter, especially the relations among the main joints of the characters, such as wrists, arms, knees, legs, waists, upper limbs, and hips. (See Figure 2 for three- dimen-
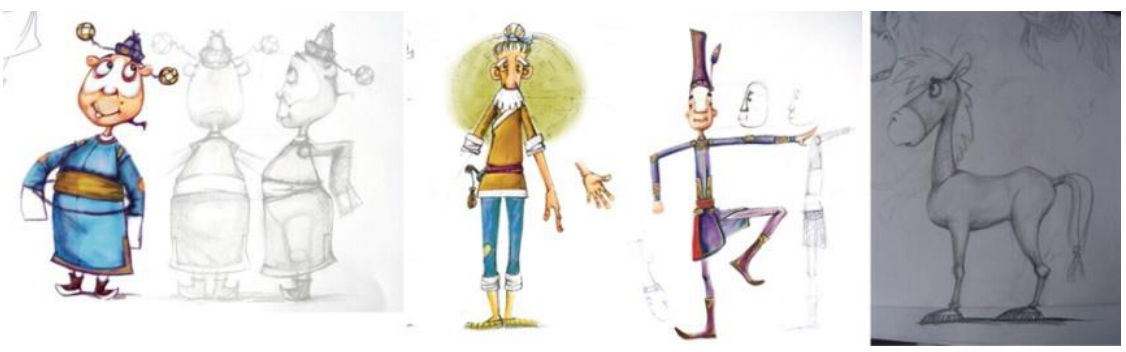

Figure 1. The character design of The Horse
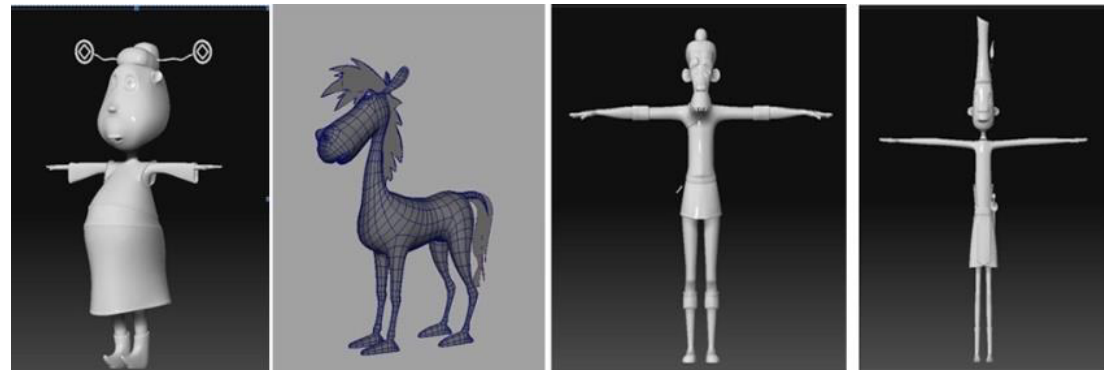

Figure 2. The three-dimensional models of The Horse 
sional models)

\subsection{UV is the key carrier of the correlation between model and color art}

UV is the attached carrier of modeling material. It is an important channel to create colors, patterns and structures in three-dimensional animation models. As shown in Figure 3, character UV is produced by LAYOUT through mapping. Two-dimensional UV can be exported from material editor and imported into Photoshop for drawing (as shown in Figure 4 and Figure 5). After the drawing is done, it can be connected to the models through preset MAYA material ball (as shown in Figure 6).

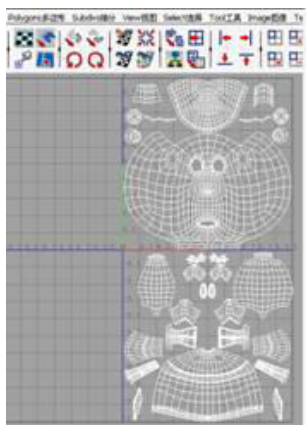

Figure 3. Character UV

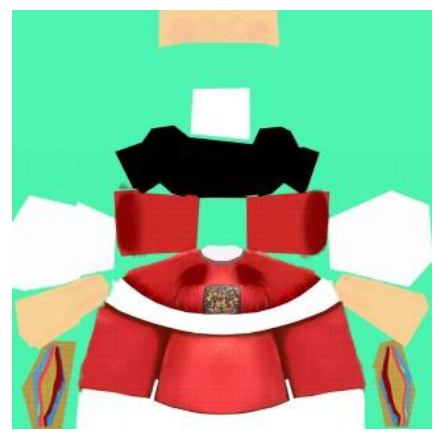

Figure 4. The body UV Mapping
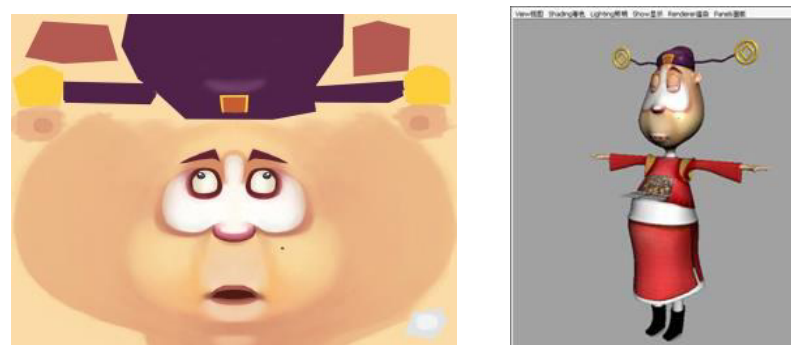

Figure 5. The body UV Mapping Figure 6. The role material

The characters' social status and personalities have been fully considered during the production of The Horse. For example, in order to show the magistrate's characteristics and clownish presentation, red is used to replace the originally considered blue as the background color of his clothes so as to better manifest the entertainment of the story. Chinese opera facial makeup is used to draw the magistrate's head while the patterns and styles of the official robes in ancient China are also considered to match the entire color scheme. In this part, Photoshop is applied in delineating, coloring, and creating clothes plication. And it can also be used to timely change the observation effects of the three-dimensional models if necessary. After the basic colored mappings are completed, highlight and bump mapping are also required for further processing. At last, the connection and adjustment of material balls are conducted in MAYA to reach the expected effect.

\subsection{Uniformity of motion modulation and performance art of three-dimensional animation character}

\subsubsection{Body constitution is the technical proof for the} skeleton setting of three-dimensional character Skeleton system is the precondition of character model motion. Binding must be strictly done according to character structure so as to avoid difficulty in producing following motions. As shown in the figure given below, take the magistrate as an example. Some condyles can be added on the waist to make his motions more natural and real. A parent skeleton is added under his hip to control the whole body. The skeletons on his feet are kind of special. They are set up for driving. After all skeletons are set up, historical records need to be deleted. It is better to name each skeleton to make it more convenient for weight brushing. The next step is to add controller which serves for motion adjustment. Take the magistrate's hands as an example. IK handle is controlled by the controller and the skeleton is controlled by IK handle. Control of three levels or more levels are set to control the hands and make it more convenient for motion adjustment as skeleton selection will make the whole process much more complicated. Key frames are set up for the feet and the hands and controllers can be used to control the hands and the feet. Historical records need to be continuously deleted during production to avoid mistake. After controllers are added, rename the locations they are in and accomplish freeze transformations of the controller attribute values. Check whether all the controllers are valid. If any mistake is found, find the cause as soon as possible as it will be much more troublesome to modify after covering. Conduct smooth bind after the check is done, and then start weight brushing. During weighting, patience and perseverance are required. Reasonable weight allocation shall be conducted in accordance with joint structures. After all the above steps are completed, character skeleton binding is done (as shown in Figure 7).
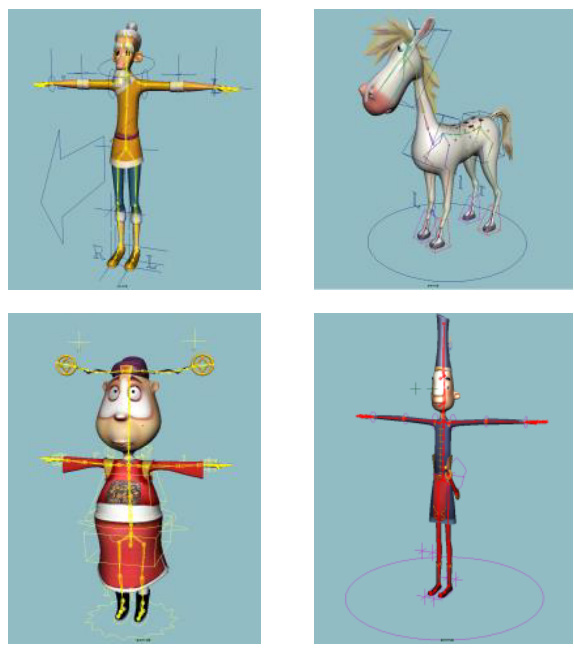

Figure 7. Diagrams of character skeleton design and binding 


\subsubsection{Animation performance is the condition of cre- ating three-dimensional motion}

Carlos Baena, an animator from the famous American animation studio PIXAR, once said, "The difference between a good animator and an animation master is like that between a good actor and an acting master." Animation performance attaches life or soul to characters or virtual models. It can make the characters or models vivid and natural. There is no doubt that animation performance is playing a key role in animation production. Just as its name implies, animation performance is to perform by cartoons which can move. Animation creators must realize the performing process with their pens in hand, and make the motion producers understand the motion rules through performance. Animation performance emphasizes on motion - mental activities are manifested through the combination of body motion, facial expressions and language. All the above constitute the foundation of three-dimensional animation motion. ${ }^{[6]}$ The production of this animation was also in accordance with such scheme. After receiving the files, the motion group checked binding and made character performance in accordance with the story. They used DV video camera to shoot or draw the motion rules; analyzed the sequences of moving rules and key gestures; completed artistic processing and shifted it to three-dimensional models. Pose-to-pose method was applied in posture production. After key frames were adjusted, middle frames were adjusted to reach the required animation effect through repeated modification. After this motion was finished, it was outputted for video preview. All previews were gathered for watch. If any failed to accord with the style of the entire animation, it would be returned for adjustment. The relation between time and interval, the moving rhythm, must be well controlled in motion adjustment. Besides, animators shall go deep into the personalities of the characters and their role statuses in the story. All these require high artistic aesthetics and creativity on animation designers.

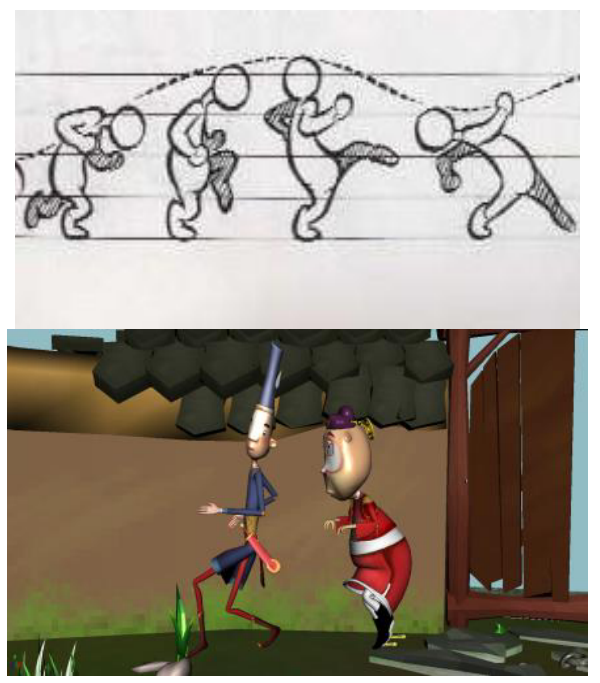

Figure 8. Characters' movements of The Horse

\subsection{Uniformity of lighting embellishment technology, picture, and space art}

Modeling and creation of frames in accordance with story line can manifest the important role that three-dimensional lighting plays. Three-dimensional lighting can light up the setting, make objects show various reflection effects, create shade and special effects, and shape beautiful and fine frames through lighting effects and materials. MAYA lighting is divided into ambient light, parallel light, floodlight, spot light, and surface light. They all have their own characteristics, but also share common features. In animation creation, lighting is alone to exist. In most cases, several kinds of lighting will be used in combination. According to RGB Illumination Rule of Addition, mixing and adjustment of hue, saturation, brightness (HSV) and Alpha value can also be realized in lighting.

Lighting is to determine light ray and direction of main light source of the setting which is similar to oil painting in which direction of main light source and major color relations of painting need to be determined. The keynote of setting light serves for information and storyline creation during story development. In addition, the empty and full relations, relations between cold tone and warm tone, and the volume relations among model objects should be considered to realize gradation changes in light ray. Lighting can also make up the defects in the mapping of objects.

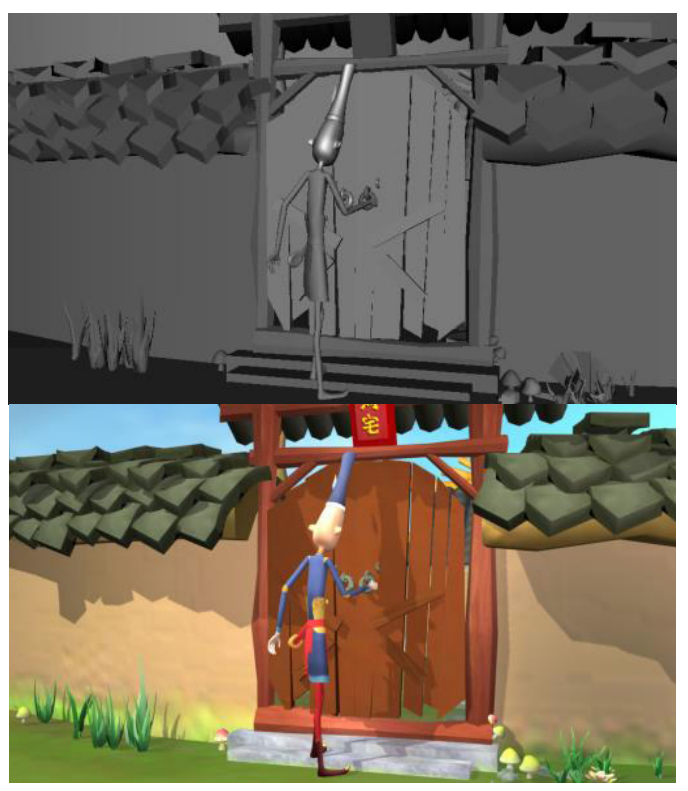

Figure 9. The still of The Horse

\section{CONCLUSIONS}

To a large extent, the perceptual and entertainment features of digital artistic aesthetics have been popularized by the comprehensive power originated from the mutual combination of contemporary digitization 
and multimedia. Maybe, this is some manifestation to reflect the self-worth and significance of digital technology under the background of art. Technology and art are the two attributes of three-dimensional animation development, and we shall be able to understand the relation between them in the right way. To catch one and lose the other will only lead to isolated and lopsided views. The application of CG technology in three-dimensional animation is not only a breakthrough in animation production technology. In a higher level, it can free animation artists from the restrictions set by realizable conditions Moreover, it can concentrate and focus the ideas, emotion, understanding and views of ourselves, others and social groups on the creation of animation characters, in order to obtain artistic breakthrough. Without animation technology as the support, artistic concept alone will be castle in the air or mirage. Of course, animation artistic expression without technology is like a tree without roots. Therefore, employees in animation industry need continuous exploration in both new technologies and new artistic expression forms to create animation works with era significance. They need to perfectly combine new technologies and new artistic expression forms; update their creative ideas; and improve manifestation patterns.

\section{ACKNOWLEDGEMENT}

The paper is funded by the Key Research Base of Humanities and Social Science in Universities of $\mathrm{Hu}$ bei "Research Centre of Tourist Economy and Culture of Dabie Mountain" (GN: 2014001903); the Research and Guide Project of Humanities and Social Science of Ministry of Education of Hubei Province (14G386).

\section{REFERENCES}

[1] Ye, S.Q. Tsung-Dao Lee's historical contribution to the combination of science and art. World Sci-tech $R \& D$, 22(2).

[2] Catalogue of BFI National Library. 2005. Nanfang Sports, B19, July $19^{\text {th }}$.

[3] Li, M. 2008. CG technology: The flying wings of animation art. Journal of Xinjiang Arts University, (3).
[4] Zhuang, Y.T., Liu, X.M. \& Pan, Y.H. 2000. A human animation skeleton extraction technique based on video. Journal of Computer Research and Development, 37(4).

[5] Wu, Z.G. \& Qi, X.B. 2010. Television Animation Performance. Anhui: University Science and Technology of China Press.

[6] Yetian, G.W. 1984. (Japan): Basis of Play Structure. World Cinema, 4.

[7] Cong, Y.H. 2010. Brief analysis of three-dimensional animation technology in view of the movie G-force. Film Literature, 23.

[8] Dang, M., Zheng, D. \& Wang, Y.Z. 2008. Analysis of the technical character and artistic quality of contemporary Chinese animation. Movie Review, 19.

[9] Wang, K. 2009. Brief discussion about the nature of animation in the animation production in digital age. Youth Liberator, 19.

[10] Zhang, Y.Y. \& Wang, X.F. 2008. Spirit of animation art in context of digital technology. Movie Review, 24.

[11] Zhao, Y.L. 2004. The technology as art - Overview of the development of three-dimensional animation. Audio \& Video Production, 7.

[12] Xu, D.P. \& Xu, Y.B. Brief analysis of current situation and development prospect of the application of three-dimensional animation art. Journal of Zhejiang Wanli University, 18.

[13] Feng, S.T. 2010. Common Shot and Shooting Scale of Flash Animation. China Computer and Communication (Theoretical Edition), 9.

[14] Ji, L. 2011. Application of non-linear editing in digital television program production. Computer Knowledge and Technology, 3.

[15] Wang, Y. 2010. Combination of montage and non-linear editing technique. Economic Research Guide, 35.

[16] Li, S.D. 2015. Outline of Digital Media Art ( $3^{\text {rd }}$ Edition). Beijing: Tsinghua University Press.

[17] Richard Williams. 2006. Survival Manual of Original Animation ( $1^{\text {st }}$ Edition). Beijing: China Youth Publishing House.

[18] Huang, Y. 2015. Combination and paradox of digital technique and artistic aesthetics in digital art. Southeast Academic Research, 5.

[19] Li, X.L. 2015. Discussion on the application of traditional aesthetic ideal in animation art. Sichuan Drama, 7.

[20] Wang, M. \& Yin, J. 2014. Brief analysis of gods and spirits designs in Japanese cartoon. Art and Design (Theory), 12.

[21] Li, Y. \& Luo, D. 2015. The enlightenment of pixar three-dimensional animation on our animation. Art Education, 10.

[22] Rong, N. \& Ma, O. 2015. Going inside and comprehending beyond-emotional expression and designs of cartoon characters. Contemporary TV, 10. 